\title{
First-Pass Contrast-Enhanced MR Angiography in Evaluation of Treated Spinal Arteriovenous Fistulas: Is Catheter Angiography Necessary?
}

\author{
(D) S. Mathur, (D) S.P. Symons, (DT.J. Huynh, DT.R. Marotta, DR.I. Aviv, and (D) A. Bharatha
}

\begin{abstract}
BACKGROUND AND PURPOSE: Catheter angiography is typically used for follow-up of treated spinal AVFs. The purpose of this study was to determine the diagnostic performance and utility of first-pass contrast-enhanced MRA in the posttreatment evaluation of spinal AVFs compared with DSA.
\end{abstract}

MATERIALS AND METHODS: A retrospective review was performed of all patients at our tertiary referral hospital (from January 2000 to April 2015) who underwent spine MR imaging, first-pass contrast-enhanced MRA, and DSA after surgical and/or endovascular treatment of a spinal AVF. Presence of recurrent or residual fistula on MRA, including vertebral level of the recurrent/residual fistula, was evaluated by 2 experienced neuroradiologists blinded to DSA findings. Posttreatment conventional MR imaging findings were also evaluated, including presence of intramedullary $\mathrm{T} 2$ hyperintensity, perimedullary serpentine flow voids, and cord enhancement. The performance of MRA and MR imaging findings for diagnosis of recurrent/residual fistula was determined by using DSA as the criterion standard.

RESULTS: In total, 28 posttreatment paired MR imaging/MRA and DSA studies were evaluated in 22 patients with prior spinal AVF and 1 patient with intracranial AVF with prior cervical perimedullary venous drainage. Six image sets of 5 patients demonstrated recurrent/ residual disease at DSA. MRA correctly identified all cases with recurrent/residual disease with 1 false-positive (sensitivity, 100\%; specificity $95 \% ; P<.001$ ), with correct localization in all cases without interobserver disagreement. Conventional MR imaging parameters were not significantly associated with recurrent/residual spinal AVF.

CONCLUSIONS: First-pass MRA demonstrates high sensitivity and specificity for identifying recurrent/residual spinal AVFs and may potentially substitute for DSA in the posttreatment follow-up of patients with spinal AVFs.

ABBREVIATION: SAVF $=$ spinal AVF

$\mathbf{S}$ pinal AVFs (SAVFs) can cause radicular/perimedullary venous reflux and present with progressive myelopathy due to cord congestion. The goal of treatment is to disconnect the refluxing vein to protect the cord from further damage. The most common vascular lesion to present in this fashion is

Received June 14, 2016; accepted after revision August 9.

From the Division of Diagnostic and Interventional Neuroradiology, Department of Medical Imaging (S.M., T.J.H., T.R.M., A.B.), St. Michael's Hospital, University of Toronto, Toronto, Ontario, Canada; and Division of Neuroradiology, Department of Medical Imaging (S.M., T.J.H., R.I.A., S.P.S.) and Department of OtolaryngologyHead and Neck Surgery (S.P.S.), Sunnybrook Health Sciences Centre, University of Toronto, Toronto, Ontario, Canada.

Paper previously presented in part at: Annual Meeting of the American Society of Neuroradiology, April 25-30, 2015; Chicago, Illinois.

Please address correspondence to Aditya Bharatha, MD, FRCPC, Department of Medical Imaging, St. Michael's Hospital, 30 Bond St, Room 3-077CC, Toronto, ON Canada M5B 1W8; e-mail: bharathaa@smh.ca

三 Indicates article with Supplemental online table.

http://dx.doi.org/10.3174/ajnr.A4971 the spinal dural AVF. However, similar clinical and radiologic appearances can occur with intracranial dural fistulas draining into the spinal venous system, epidural fistulas with intrathecal venous reflux as well as perimedullary and filum terminale fistulas. Prevalence of recurrent or residual fistulas after treatment of SAVFs ranges from $3.4 \%$ to $27.8 \%$ and is associated with progressive myelopathy and morbidity. ${ }^{1}$ Fistula recurrence may occur early within 1 month after treatment or present in delayed fashion years after successful treatment. ${ }^{1}$ Conventional spine MR imaging findings of SAVF, including perimedullary flow voids, intramedullary T2 hyperintensity, and cord enhancement, are not reliable markers of residual/ recurrent fistula. ${ }^{2,3}$ Using clinical symptoms alone to assess for residual or recurrent disease may result in delayed diagnosis and irreversible progression of symptoms. ${ }^{4}$ Therefore, posttherapy evaluation of patients with previously treated SAVF is commonly performed to ensure complete fistula occlusion. 
DSA remains the criterion standard test; however, it is an invasive test associated with some procedural risks. ${ }^{5}$ Spine MRA may be a useful noninvasive tool for initial posttreatment evaluation of SAVFs and may have the potential to be a substitute for DSA for this indication. ${ }^{3,4,6}$ In this study, we evaluated the performance of MRA for identifying recurrent/residual SAVF posttreatment, compared with DSA and conventional MR imaging findings.

\section{MATERIALS AND METHODS}

\section{Patients}

After institutional ethics board approval, we retrospectively reviewed all consecutive patients (from January 2000 to April 2015) who underwent spine MR imaging, including MRA and DSA at our institution (St. Michael's Hospital). Patients were included in the study if they had imaging as part of follow-up of a treated SAVF (including spinal dural, epidural, perimedullary, and filum terminale AVFs) and/or for suspected residual/recurrent SAVF posttreatment. At our tertiary care referral hospital, patients routinely have DSA after SAVF treatment to confirm absence of residual fistula. MR imaging/MRA is typically performed at 1-6-month follow-up, depending on neurosurgeon and/or protocolling neuroradiologist preference, or earlier if there is clinical concern, with subsequent DSA performed as clinically indicated. There were no treatments for SAVFs between the MR imaging/MRA and DSA studies. For the purposes of this study, MR imaging/MRA studies were compared with the DSA performed closest to the date of the MR imaging/MRA study. Patient demographics and days between MR imaging/MRA, DSA, and treatments were retrieved from retrospective chart review.

Note: Some study subjects from our data base have been included in another research paper testing a different research question ("First-Pass Contrast-Enhanced MR Angiography for Pretherapeutic Diagnosis of Spinal Epidural Arteriovenous Fistulas with Intradural Venous Reflux," also in this issue).

\section{MR Imaging and MRA Technique}

All MR imaging and MRA studies were performed on a $1.5 \mathrm{~T}$ system (Intera Achieva; Philips Healthcare, Best, the Netherlands) by using a dedicated 5-channel spine coil in supine position. Conventional whole-spine MR imaging sequences included sagittal T2WI, sagittal T1WI, axial T2WI, and postcontrast sagittal and axial T1WI.

First-pass MRA was performed by using a manually triggered timed-delay technique. Three-plane spine localizers were obtained, and the sagittal plane was selected for imaging. The FOV was $33 \mathrm{~cm}$ craniocaudally, and imaged location was selected by a neuroradiologist based on the location of the treated SAVF. Studies were performed by using gadolinium-based contrast agents including Omniscan (gadodiamide; GE Healthcare, Piscataway, New Jersey) or, more recently, MultiHance (gadobenate dimeglumine; Bracco Diagnostics, Princeton, New Jersey). Acquisition delay time was determined by using a 2 -mL IV contrast test bolus, and peak enhancement of the abdominal aorta was measured by using 2D MR fluoroscopy. This was followed by intravenous injection of $18 \mathrm{~mL}$ of contrast agent at $2 \mathrm{~mL} / \mathrm{s}$ injection rate by using an MR compatible power injector (Spectris; Medrad, Indianola, Pennsylvania) followed by $20-\mathrm{mL}$ saline bolus. This imaging pro- tocol was used for all patients. Manually triggered, single-phase, $3 \mathrm{D}$ acquisition was performed with scan parameters as follows: $400 \times 512$ matrix, $0.82 \times 1.08 \mathrm{~mm}$ in-plane resolution reconstructed to $0.64 \times 0.64 \mathrm{~mm}$ with $0.9-\mathrm{mm}$ section thickness, $\mathrm{TR}=$ $5.4 \mathrm{~ms}, \mathrm{TE}=1.76 \mathrm{~ms}$, flip angle $=300, \mathrm{NEX}=1$, overcontiguous sections, and scan time of 47 seconds. Background subtracted image sets with multiplanar MIP images were obtained by automated postprocessing.

\section{DSA Technique}

A dedicated biplanar neuroangiographic system (Artis; Siemens, Erlangen, Germany) was used for the spinal DSA examinations. A femoral approach was used under general or local anesthesia. Iodinated contrast agent (Omnipaque 300; GE Healthcare) was selectively injected bilaterally into segmental arteries at the level of the treated fistula and also at least 2 vertebral levels above and below the site of the fistula. Magnification, oblique and high frame rate angiography were used, as appropriate. 3D DSA was occasionally used to define the angioarchitecture. If the site of treated fistula was unclear, angiographers were encouraged to inject into the site suspected for fistula on MRA, and if unsuccessful, complete spinal angiography was performed.

\section{Imaging Analysis}

Two experienced neuroradiologists (S.P.S. and A.B.) with 13 and 7 years of experience, respectively, reviewed all posttreatment MR imaging and MRA studies. Readers were blinded to DSA findings and diagnosis, but had access to pretreatment MR imaging and MRA images while reviewing the studies. For MRA studies, the readers noted the presence or absence of an SAVF and the level and side of the fistula if present. The presence of a residual/recurrent fistula was based on the visualization of arterially enhancing prominent intradural veins on the source images of the MRA sequence. The level and side of the fistula were determined on MRA by looking for the point of fistulization (commonly seen as a small tuft of vessels in the foraminal region) and for the level at which the proximal part of the draining intradural (radicular) vein appeared. For conventional MR imaging studies, readers were asked to evaluate pre- and posttreatment MR imaging findings, including presence or absence of 1) intramedullary T2 hyperintensity, 2) perimedullary serpentine flow voids, and 3) cord enhancement. After making their observations on MR imaging and MRA studies, the readers reviewed the DSA images for presence or absence of SAVF and noted the level and side of fistula if present.

\section{Statistical Analysis}

For descriptive statistics, categoric variables are presented as percentages, and continuous variables are presented as medians with interquartile range. Diagnostic performances with 95\% CI of MRA and MR imaging were calculated by using DSA as the reference standard. Fisher exact and $\chi^{2}$ tests were used to identify associations for categoric data where appropriate. Statistical significance was defined as $P<.05$ for all tests. Statistical analyses were performed by using MedCalc for Windows, version 12.5 (MedCalc Software, Mariakerke, Belgium) and R, version 3.1.1 (R Foundation, http://www.r-project.org/).

AJNR Am J Neuroradiol 38:200-05 Jan 2017 www.ajnr.org 


\section{RESULTS}

In total, 23 patients (median age, 62 years; interquartile range, 54-69 years; 19 male [83\%]) fulfilled the study inclusion criteria. Five patients had 2 sets of MR imaging/MRA and DSA studies, yielding a total of 28 paired posttreatment studies. The median time (interquartile range) interval between treatment and MRA, MRA and DSA, and treatment and DSA was 80 (11-277) days, 53 (2-122) days, and 3 (1-20) days, respectively. Of the 23 patients with AVF, there were 16 spinal dural AVFs, 4 spinal epidural AVFs, 1 perimedullary AVF, 1 filum terminale fistula, and 1 patient with an intracranial dural AVF, all presenting with perimedullary venous congestion and myelopathy.

All patients underwent surgical treatment of the fistula, aside from 1 patient in whom a combined endovascular and surgical procedure was performed. Previously treated AVF levels were at C4 $(n=1), \mathrm{C} 7(n=1), \mathrm{T} 5(n=2), \mathrm{T} 9(n=4), \mathrm{T} 12(n=4), \mathrm{L} 1$ $(n=1), \mathrm{L} 2(n=2), \mathrm{L} 3(n=3), \mathrm{L} 4(n=2), \mathrm{S} 5(n=1)$, filum terminale $(n=1)$, and intracranial $(n=1)$.

\section{Diagnosis and Localization of Residual or Recurrent SAVF on MRA}

There were 6 image sets of residual/recurrent SAVF in 5 patients, which are summarized in the On-line Table. All 6 (100\%) cases of residual/recurrent SAVF were correctly identified on MRA by the 2 study readers as identified by arterial phase perimedullary venous enhancement within enlarged intradural perimedullary vessels (Fig 1). Of 22 cases without residual/recurrent fistula at DSA, 21 of $22(95 \%)$ were correctly identified on MRA to not demonstrate a residual/recurrent fistula (Fig 2). One of the 22 (5\%) cases demonstrated a false-positive on MRA, as demonstrated by subtle enhancement of perimedullary veins thought to be abnormal, but no shunt was present at follow-up comprehensive DSA (Fig 3). All available pretreatment MRA identified the fistula. Pretreatment MRA for 4 patients was not available (3 positive for residual/ recurrent disease and the false-positive case). The readers were still able to accurately distinguish residual/recurrent shunts from cured lesions (except for the 1 false-positive case) based on presence/absence of arterialized perimedullary veins. Overall sensitivity and specificity of MRA compared with DSA were $100 \%$ (95\% CI, 42\%-100\%) and 95\% (95\% CI, 77\%-100\%), respectively. Localization of the recurrent/residual fistula was correct in $100 \%$ of the cases. On MRA, there was no interobserver disagreement related to the presence or localization of residual/recurrent SAVF.

\section{Conventional MR Imaging Findings}

Pretreatment MR imaging was unavailable in 3 of the patients with residual/recurrent fistulas. Four pre- and posttreatment MR imaging datasets were available for comparison for patients with recurrent/residual AVF. All cases had pretreatment perimedullary flow voids, of which $3(75 \%)$ remained stable at posttreatment MR imaging and 1 (25\%) showed decrease. Two of 4 cases demonstrated cord edema and enhancement pretreatment, of which 1 demonstrated stability posttreatment and 1 showed mild reduction.

Pre- and posttreatment MRIs were available for review for 21 cases negative for recurrent/residual AVF on DSA (at the median

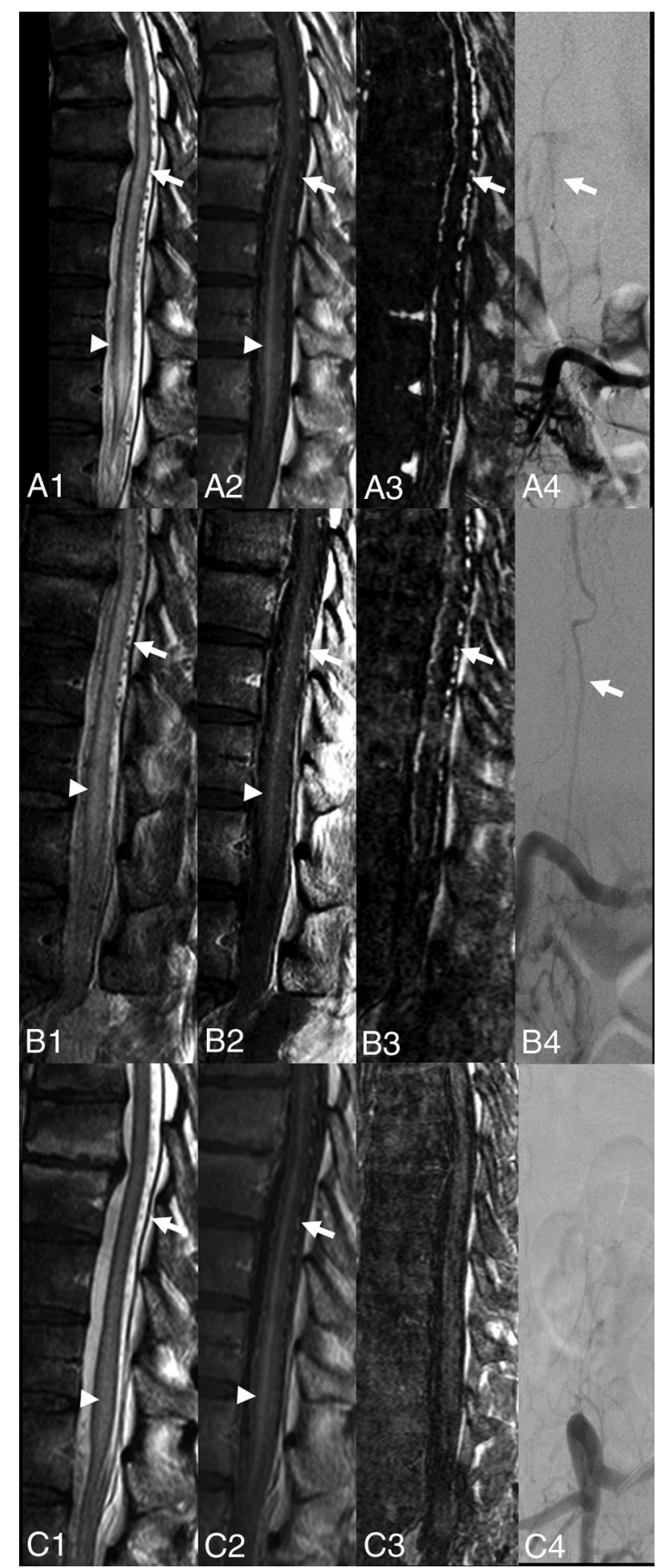

FIG 1. Spinal epidural AVF with intradural venous drainage (patient 4). Sagittal T2, postgadolinium T1, MRA MIP, and frontal DSA images pretreatment (Al-4), after first attempted surgical disconnection (BI-4), and after second surgical disconnection (Cl-4), respectively. Pretreatment study shows T2 cord hyperintensity and enhancement (A1-2, arrowheads) with surrounding enhancing perimedullary flow voids (Al-2, arrows). MRA shows arterial enhancement of intradural veins $(A 3$, arrow). DSA confirmed the $\operatorname{AVF}(A 4$, arrow indicates the arterialized radicular vein). Postsurgical study after attempted AVF disconnection (B1-4) shows findings unchanged compared with the pretreatment study, consistent with residual AVF. Second postsurgical study shows persistent $\mathrm{T} 2$ cord hyperintensity and enhancement (Cl-2, arrowheads) with decreased size of perimedullary veins (Cl-2, arrows). MRA and DSA (C3-4) confirm absence of arterialized intradural veins, consistent with a successful surgical disconnection. 


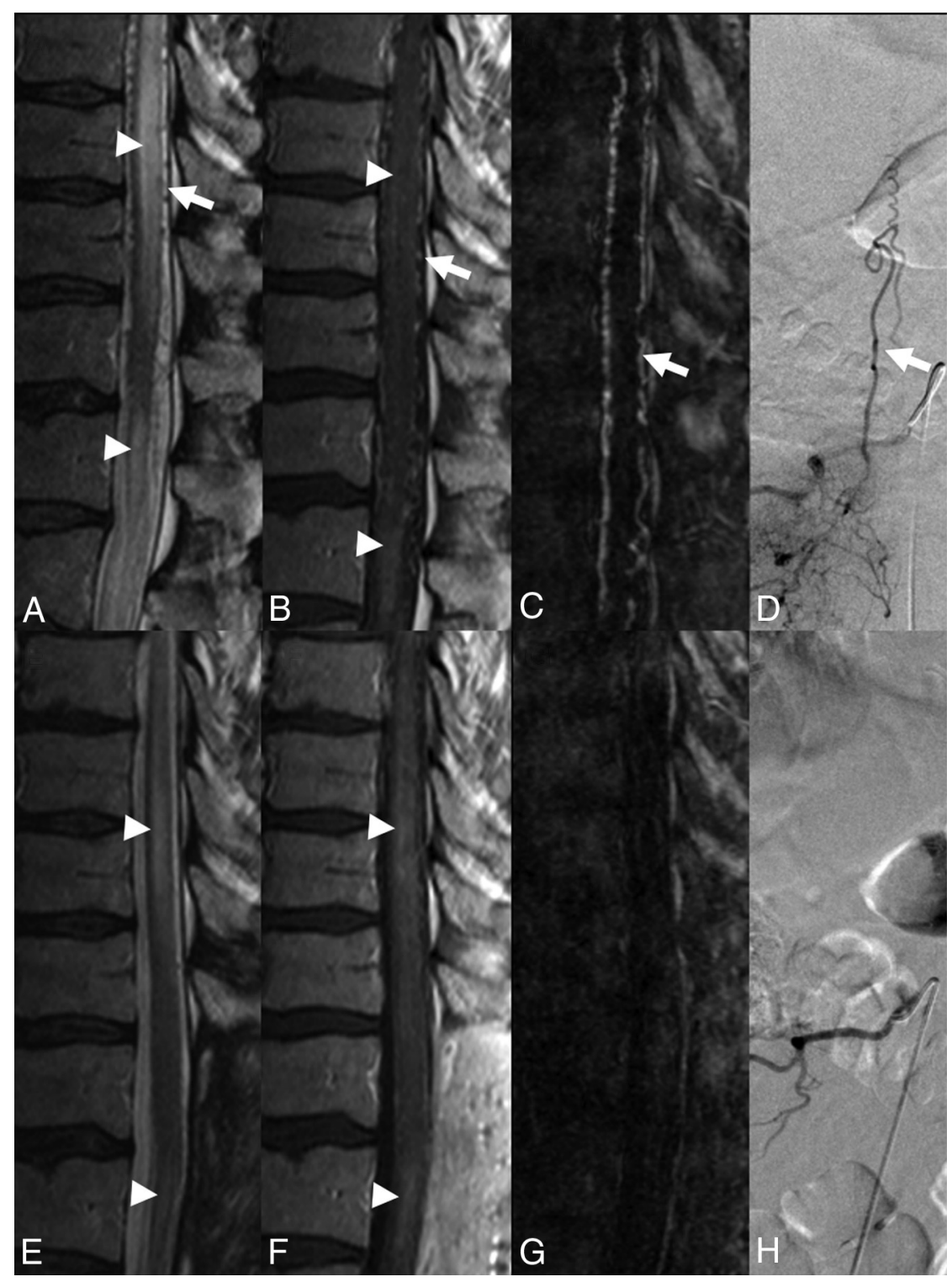

FIG 2. Spinal dural AVF in a 50-year-old man with 6 months of progressive lower extremity paraplegia. Pretreatment sagittal T2 $(A)$ and postcontrast $\mathrm{Tl}(B)$ show abnormal perimedullary vessels $(A$, arrow), which enhance postcontrast ( $B$, arrow), in addition to intramedullary $T 2$ hyperintensity ( $A$, arrowheads) with mild patchy intramedullary enhancement ( $B$, arrowheads). Pretreatment sagittal MRA MIP (C) demonstrates arterially enhancing perimedullary veins ( $C$, arrow). Right $\mathrm{L} 1$ segmental artery injection-frontal projection DSA demonstrates retrograde drainage into radicular vein $(D$, arrow). After surgical disconnection, there is reduction in intramedullary $T 2$ hyperintensity (E, arrowheads) and perimedullary flow voids, with persistent cord enhancement ( $F$, arrowheads) and lack of arterially enhancing perimedullary veins on MRA ( $G$ ). Successful fistula disconnection was confirmed on repeat DSA $(H)$.

follow-up time of 80 days). All of these had perimedullary flow voids, cord edema, and enhancement on pretreatment MR imaging. After successful treatment, $10(47.6 \%)$ showed decrease, 1 (4.8\%) showed stability, and 10 (47.6\%) showed absence of perimedullary flow voids. The cord edema decreased in 17 (81\%) cases, remained stable in $2(9.5 \%)$, and completely resolved in 2 (9.5\%). The cord enhancement decreased in 10 (48\%) cases and remained stable in the remaining 11 (52\%).

There was significant overlap in the conventional MR imaging appearances of successfully and unsuccessfully treated spinal fistulas. None of the conventional MR imaging parameters were significantly associated with recurrent/residual AVF (all $P>.05)$.

\section{DISCUSSION}

Our results provide further evidence of the utility of MRA in the posttreatment evaluation of patients with SAVFs. ${ }^{2,3,7,8}$ All cases of recurrent/residual SAVF in our study were confirmed by DSA and demonstrated abnormal arterial enhancement of perimedullary serpentine vessels on MRA (sensitivity of 100\%). Both readers correctly identified the level of the fistula, including the level of the recurrent/residual fistula, without interobserver disagreement. One false-positive study was noted on MRA (specificity of $95 \%$ ), which was primarily due to poor timing of the acquisition relative to the contrast bolus, resulting in venous contamination and enhancement of normal draining perimedullary veins mimicking arterialized veins. In that particular case, further review of 


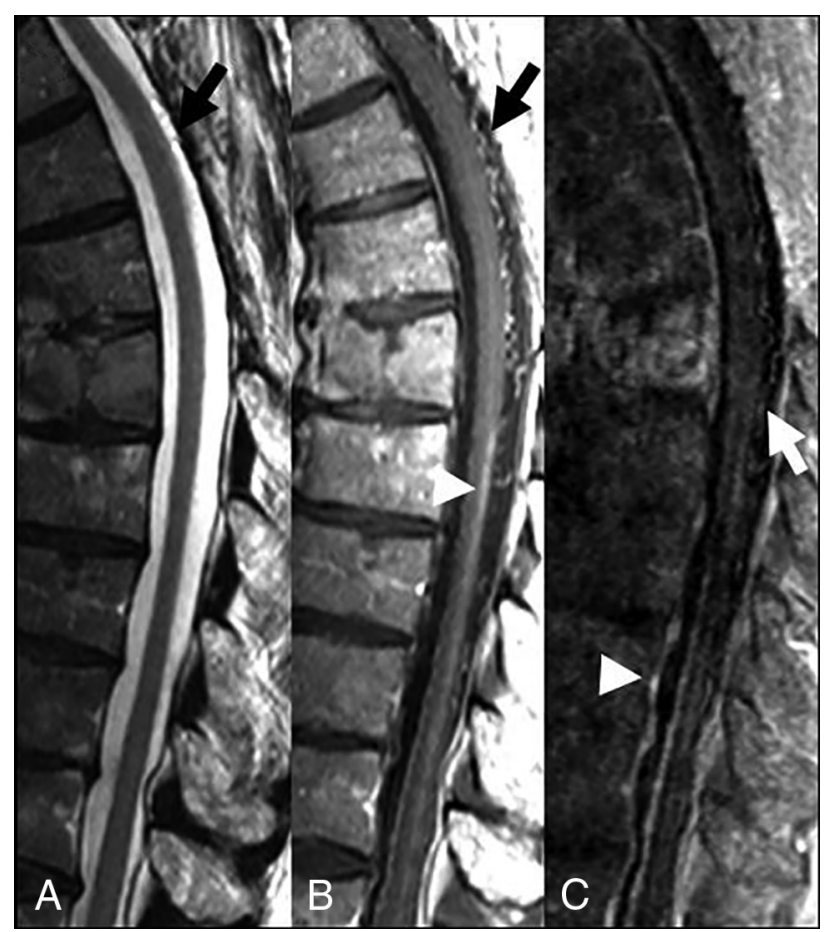

FIG 3. False-positive on MRA. 78-year-old man with prior surgical disconnection of a spinal dural AVF at left T5 level presented with worsening gait spasticity confounded by history of Parkinson disease. Few dorsal perimedullary flow voids were noted on the sagittal T2 ( $A$, arrow), which enhanced on the postcontrast T1 study ( $B$, arrow). Mild intramedullary enhancement was also noted on the postcontrast $\mathrm{T} 1$ ( $B$, arrowhead). On MRA ( $C$ ), there was faint enhancement of the perimedullary veins (C, arrow), which led to the suspicion of residual/ recurrent fistula. No fistula was found at subsequent DSA (not shown). The findings on MR imaging and MRA were presumed to be residual changes from prior fistula. Mild enhancement of the perimedullary veins may be a result of venous contamination of contrast bolus, as suggested by enhancement of the basivertebral veins ( $C$, arrowhead).

the first-pass MRA source images revealed diffuse enhancement of the epidural veins at all levels, indicating that the study was timed too late, into the venous phase, likely causing the falsepositive diagnosis. If this finding is observed, it should prompt consideration of a repeat study with strict attention to arterial phase timing. Apart from this single case, no other study was affected by contrast timing problems, indicating that the technique is generally robust.

The results of our study are consistent with previous MRA studies evaluating patients post-SAVF treatment. ${ }^{2,3,7}$ Mascalchi et $\mathrm{al}^{2}$ demonstrated the ability of 2D and 3D phase-contrast MRA and 3D time-resolved MRA to correctly identify residual/recurrent fistula in 7 of 30 patients post-SAVF treatment (MRA sensitivity of $100 \%)$. In their study, however, not all patients with negative MRA were evaluated with DSA; therefore, the diagnostic performance of MRA cannot be assessed from their study. They reported 1 patient with progressive myelopathy 2 months after an initial negative MRA study, which was later proved to be a recurrent/residual fistula at DSA. This recurrence may have represented an MRA false-negative, reducing sensitivity, and the authors hypothesize that may have been the result of insufficient flow sensitivity or spatial resolution of their MRA technique. ${ }^{2}$ Our technique used first-pass contrast-enhanced MRA, which has a higher spatial resolution than current time-resolved methods. Kaufmann et $\mathrm{al}^{7}$ evaluated 8 of 34 patients post-SAVF treatment with manually triggered first-pass MRA. One of 8 patients showed mild increased perimedullary vascularity on MRA, but was found to be negative at DSA, consistent with a false-positive. A separate patient showed moderate increased perimedullary vascularity on MRA, which was later proved positive for recurrence at DSA. MRA was normal in the remaining 6 patients, of whom 2 were confirmed DSA negative. Ali et $\mathrm{al}^{3}$ described agreement between time-resolved MRA and DSA in their series of 3 patients postSAVF treatment, 1 of whom had a recurrence. An advantage of our current study is that it is the largest series in which all patients with or without residual/recurrent SAVF on posttreatment MRA also had DSA as the criterion standard. All patients also had a uniform MRA protocol; specifically, a first-pass, gadolinium-enhanced, manually triggered $3 \mathrm{D}$-acquisition technique.

Our findings together with the reported literature suggest high sensitivity and specificity of MRA for detection of residual/recurrent fistulas, which is likely improved with modern MRA techniques. ${ }^{9,10}$ High sensitivity in particular is needed, given the screening nature of the study with use of DSA to confirm the findings and evaluate the precise angioarchitecture of the residual lesion. Ensuring consistent arterial phase acquisition of first-pass MRA without venous contamination is important and remains a potential limitation of first-pass MRA that may result in decreased specificity of first-pass MRA, as demonstrated by our false-positive and possibly by the case from Kaufmann et al. ${ }^{7}$ Use of timeresolved MRA may help improve specificity; however, there remains a balance between the need for temporal resolution needed to identify true arterial enhancement versus the spatial resolution needed to visualize and identify the level of the fistula. ${ }^{3}$

Similar to previous studies evaluating post-SAVF treatment conventional MR imaging findings, , $6,7,11$ we noted that none of the conventional MR imaging features demonstrated statistical significance for predicting recurrent/residual SAVF. Several studies have noted that intramedullary T2 hyperintensity usually reduces or resolves within months to years after treatment; however, it may be persistent after successful treatment. ${ }^{6,12}$ Similar findings may be seen with cord enhancement and perimedullary flow voids. ${ }^{2,6,7,11}$ Although improvement or resolution of conventional MR imaging findings may be associated with successful treatment, these findings may take time to resolve, and none are accurate enough to exclude residual/recurrent fistula., ${ }^{2,13}$ In contrast, our study demonstrates that MRA may more directly identify residual/recurrent disease either immediately after treatment or on follow-up. MRA may also identify enhancing perimedullary vessels not apparent on standard sagittal T2 sequences. ${ }^{7}$

A recent meta-analysis of 35 post-SAVF treatment studies including 1112 patients demonstrated a $96.6 \%$ and $72.2 \%$ initial fistula occlusion for patients treated with surgical and endovascular methods, respectively. ${ }^{1}$ Recurrences may be early (ie, $<1$ month) or late (up to several years). Willinsky et al ${ }^{6}$ hypothesized in 1995 that with advances in MRA, DSA may not be required for posttreatment follow-up of SAVFs. The high sensitivity of MRA for recurrent/residual disease in our study lends further support to this concept. Presently, MRA facilitates and does not replace DSA. Our study suggests MRA may potentially reduce the need 
for DSA in follow-up of treated fistulas for most patients who do not have residual/recurrent disease. If a residual fistula is suspected on postoperative MRA, DSA will be required, but may be facilitated by allowing a more targeted study with fewer injections aimed at the predicted level of the lesion on MRA, which may occur at a different level from the original location. This approach may potentially enable reduction of DSA procedural and fluoroscopic times and volume of contrast administered and further reduces the risks associated with potentially lengthy complete spinal angiographic examinations. 5,6,14

This study is primarily limited by a relatively small sample size, particularly with the limited number of recurrent/residual fistula; however, this is not unexpected given the rare nature of the disease. The retrospective nature of this study may also have resulted in a selection bias. Further prospective study of MRA in posttreatment patients with SAVF would be beneficial.

\section{CONCLUSIONS}

First-pass contrast-enhanced MRA demonstrates high sensitivity and specificity for identifying recurrent/residual SAVF and may potentially substitute for DSA in the posttreatment follow-up of patients with SAVF; however, prospective confirmation in a larger study is needed.

Disclosures: Thomas Marotta-UNRELATED: Consultancy: Pipeline, Comments: proctor; Patents (planned, pending or issued): Evasc Medical Systems, Comments: eCLIPS; Stock/stock options: Evasc Medical Systems, Comments: eCLIPS.

\section{REFERENCES}

1. Bakker NA, Uyttenboogaart M, Luijckx GJ, et al. Recurrence rates after surgical or endovascular treatment of spinal dural arteriovenous fistulas. Neurosurgery 2015;77:137-44; discussion 144 CrossRef Medline

2. Mascalchi M, Ferrito G, Quilici N, et al. Spinal vascular malformations: MR angiography after treatment. Radiology 2001; 219:346-53 CrossRef Medline
3. Ali S, Cashen TA, Carroll TJ, et al. Time-resolved spinal MR angiography: initial clinical experience in the evaluation of spinal arteriovenous shunts. AJNR Am J Neuroradiol 2007;28:1806-10 CrossRef Medline

4. Brinjikji W, Nasr DM, Morris JM, et al. Clinical outcomes of patients with delayed diagnosis of spinal dural arteriovenous fistulas. AJNR Am J Neuroradiol 2016;37:380-86 CrossRef Medline

5. Forbes G, Nichols DA, Jack CR Jr, et al. Complications of spinal cord arteriography: prospective assessment of risk for diagnostic procedures. Radiology 1988;169:479-84 CrossRef Medline

6. Willinsky RA, terBrugge K, Montanera W, et al. Posttreatment MR findings in spinal dural arteriovenous malformations. AJNR Am J Neuroradiol 1995;16:2063-71 Medline

7. Kaufmann TJ, Morris JM, Saladino A, et al. Magnetic resonance imaging findings in treated spinal dural arteriovenous fistulas: lack of correlation with clinical outcomes. J Neurosurg Spine 2011;14: 548-54 CrossRef Medline

8. Condette-Auliac S, Boulin A, Roccatagliata L, et al. MRI and MRA of spinal cord arteriovenous shunts. J Magn Reson Imaging 2014;40: 1253-66 CrossRef Medline

9. Amarouche M, Hart JL, Siddiqui A, et al. Time-resolved contrastenhanced MR angiography of spinal vascular malformations. $A J N R$ Am J Neuroradiol 2015;36:417-22 CrossRef Medline

10. Lindenholz A, TerBrugge KG, van Dijk JM, et al. The accuracy and utility of contrast-enhanced MR angiography for localization of spinal dural arteriovenous fistulas: the Toronto experience. Eur Radiol 2014;24:2885-94 CrossRef Medline

11. Song JK, Viñuela F, Gobin YP, et al. Surgical and endovascular treatment of spinal dural arteriovenous fistulas: long-term disability assessment and prognostic factors. J Neurosurg Spine 2001;94:199-204 CrossRef

12. Horikoshi T, Hida K, Iwasaki Y, et al. Chronological changes in MRI findings of spinal dural arteriovenous fistula. Surg Neurol 2000;53: 243-49 CrossRef Medline

13. Morris JM. Imaging of dural arteriovenous fistula. Radiol Clin North Am 2012;50:823-39 CrossRef Medline

14. Luetmer PH, Lane JI, Gilbertson JR, et al. Preangiographic evaluation of spinal dural arteriovenous fistulas with elliptic centric contrast-enhanced MR angiography and effect on radiation dose and volume of iodinated contrast material. AJNR Am J Neuroradiol 2005; 26:711-18 Medline 